\title{
Microsatellite alterations at selected tetranucleotide repeats are associated with morphologies of colorectal neoplasias
}

\author{
Sun-Young Lee ${ }^{*} \dagger$, Heekyung Chung ${ }^{\dagger}$, Bikash Devaraj ${ }^{\dagger}$, Moriya Iwaizumi ${ }^{\dagger}$, Hye Seung \\ Han $\ddagger$, Dae-Yong Hwang, MD§, Moo Kyung Seong, MD§, Barbara H. Jung, MD $†$, and John M. \\ Carethers, MD ${ }^{\dagger}, \emptyset$ \\ "Department of Internal Medicine, Konkuk University School of Medicine, Seoul, Korea \\ ¥Department of Pathology, Konkuk University School of Medicine, Seoul, Korea \\ §Department of Surgery, Konkuk University School of Medicine, Seoul, Korea \\ tDepartment of Medicine and Moores Comprehensive Cancer Center, University of California San \\ Diego \\ IDepartment of Internal Medicine, University of Michigan
}

\begin{abstract}
Background \& Aims-Elevated microsatellite alterations at selected tetranucleotide repeats (EMAST) occurs during microsatellite instability (MSI) that is not associated with major defects in DNA mismatch repair (MMR) but rather the reduced (heterogenous) expression of the MMR protein hMSH3; it occurs in sporadic colorectal tumors. We examined the timing of development of EMAST during progression of colorectal neoplasias and looked for correlations between EMAST and clinical and pathology features of tumors.
\end{abstract}

\begin{abstract}
Methods-We evaluated tumor samples from a cohort of patients that had 24 adenomas and 84 colorectal cancers. EMAST were analyzed after DNA microdissection of matched normal and tumor samples using the polymorphic tetranucleotide microsatellite markers MYCL1, D9S242, D20S85, D8S321, and D20S82; data were compared with clinical and pathology findings. Traditional MSI analysis was performed and hMSH3 expression was measured.
\end{abstract}

\begin{abstract}
Results-Moderately-differentiated adenocarcinomas and poorly-differentiated adenocarcinomas had higher frequencies of EMAST (56.9\% and $40.0 \%$, respectively) than well-differentiated adenocarcinomas $(12.5 \%)$ or adenomas $(33.3 \%)(P=0.040)$. In endoscopic analysis, ulcerated tumors had a higher frequency of EMAST $(52.3 \%)$ than flat $(44.0 \%)$ or protruded tumors $(20.0 \%)(P=0.049)$. In quantification, all tumors with $>3$ tetranucleotide defects lost MSH3 ( $>75 \%$ of cells); nuclear
\end{abstract}

\footnotetext{
(C) 2010 The American Gastroenterological Association. Published by Elsevier Inc. All rights reserved

Correspondence to: John M. Carethers Department of Medicine University of Michigan 1500 E. Medical Center Drive, SPC 5368 Ann Arbor, MI 48109-5368 Tel: 734-936-4495 Fax: 734-232-3838 jcarethe@umich.edu.

Contribution of authors: Sun-Young Lee - Collected samples as an endoscopist at Konkuk University, conducted experiments \& study design at UCSD, and wrote manuscript

Heekyung Chung, Bikash Devaraj, Moriya Iwaizumi, and Barbara H. Jung -conducted experiments at UCSD

Hye Seung Han - Analyzed samples as a pathologist at Konkuk University

Dae-Yong Hwang and Moo Kyung Seong - Collected samples as colorectal surgeons at Konkuk University

John M. Carethers - Study design and wrote manuscript

Publisher's Disclaimer: This is a PDF file of an unedited manuscript that has been accepted for publication. As a service to our customers we are providing this early version of the manuscript. The manuscript will undergo copyediting, typesetting, and review of the resulting proof before it is published in its final citable form. Please note that during the production process errors may be discovered which could affect the content, and all legal disclaimers that apply to the journal pertain.

There are no conflicts of interest for any authors on this manuscript.
} 
heterogeneity of hMSH3 occurred more frequently in EMAST-positive $(40.0 \%)$ than in EMASTnegative tumors $(13.2 \%)(\mathrm{P}=0.010)$.

Conclusions-EMAST is acquired during progression of adenoma and well-differentiated carcinomas to moderately and poorly differentiated carcinomas; it correlates with nuclear heterogeneity for hMSH3. Loss of hMSH3 corresponds with multiple tetranucleotide frameshifts. The association between EMAST and ulcerated tumors might result from increased inflammation.

\section{Keywords}

Colon cancer; mutation; DNA repair; cancer genetics

\section{INTRODUCTION}

Defects in DNA mismatch repair (MMR) cause microsatellite instability (MSI) and help direct subsequent development of malignancy in patients with Lynch syndrome (due to germline mutation of a DNA MMR gene), and in 15-20\% of patients with sporadic colorectal cancers (CRC) (due to epigenetic inactivation of the DNA MMR gene hMLH1). ${ }^{1-4}$ Loss of DNA MMR in these conditions manifest as frameshift mutations in sequences that contain mono-[e.g. $(A)_{n}$ ] or di-nucleotide [e.g. $(\mathrm{CA})_{n}$ ] repeats that loop during DNA replication. A separate form of MSI described in respiratory, genitourinary, and other tumors, ${ }^{5-12}$ and previously not linked to defects in DNA MMR is termed elevated microsatellite alterations at selected tetranucleotide repeats (EMAST). EMAST is identified when tetranucleotide DNA sequences [e.g. $(\mathrm{AAAG})_{\mathrm{n}}$ or $(\mathrm{CTTT})_{\mathrm{n}}$ ] show frameshift mutations. In both MSI and EMAST alterations, frameshifts occur by the length of the repeat unit due to the nature of complimentary binding with the newly-synthesized strand. Most microsatellites are in non-coding region of DNA, but a small minority resides in genes that may drive the pathogenesis of CRC in particular, as frameshifts in these genes have functional growth consequences for cells. ${ }^{2,13}$

In a sentinel report, EMAST was observed in $60 \%$ of CRC and associated with heterogenous expression of the DNA MMR protein hMSH3, suggesting a causal link between loss of the hMSH3 repair profile and EMAST. While all classic MSI tumors in this report also demonstrated EMAST, most EMAST tumors were not MSI, indicating that EMAST spans multiple forms of genomic instability, and may be an acquired defect. Indeed, it has been suggested that an overlapping mechanism may exist between MSI-low (MSI-L), EMAST and loss of heterozygosity (LOH),${ }^{14}$ as all of these mechanisms have not been associated with inactivation of DNA MMR. The observation of EMAST in CRC begs the question of its timing for occurrence in the adenoma-carcinoma sequence given its finding in $60 \%$ of colorectal cancers, and what clinicopathological patterns might predict its presence. The linkage of timing and clinicopathological data to EMAST may indicate the consequences of EMAST upon tumor development while its cause is being evaluated. We therefore examined EMAST in adenomas and various staged adenocarcinomas to assess timing during colorectal neoplastic progress and correlated associated clinicopathological data with EMAST.

\section{MATERIALS and METHODS}

\section{Tissue samples}

We evaluated a cohort of 108 patients who have 24 adenomas and 84 colorectal cancers that were resected between 2008 and 2009 at Konkuk University Medical Center, Seoul, South Korea. All patients provided informed consent, and this study was approved by the Institutional Review Board of Konkuk University Medical Center, which confirmed that the study was in accordance with the ethical guidelines of the Helsinki Declaration. This study was also 
approved by the Institutional Review Board on Human Subjects Protection at University of California San Diego.

Location, size, and shape of tumors were recorded at colonoscopy. Macroscopic features were divided into three major types: (i) protruded, elevated, or polypoid, (ii) sessile, superficial, or flat, and (iii) ulceroinfiltrative, depressed, or excavated (Figure 1). Tumors showing multiple macroscopic features were classified on the predominant subtype. When the tumor was suspected or diagnosed as adenoma, endoscopic resection was done by an endoscopist (Dr. S.Y. Lee). When the tumor was suspected or diagnosed as cancer, it was referred for surgical resection by a surgeon (Dr. D.-Y. Hwang or M. K. Seong).

All specimens were diagnosed by a single pathologist (Dr. H. S. Han) to avoid interpersonal variability. Histopathology, width, height, and depth of the tumor were recorded. To differentiate between well-differentiated and moderately-differentiated adenocarcinomas, the following diagnostic criteria was used: (i) well-differentiated adenocarcinomas were diagnosed when gland-forming area encompasses more than $95 \%$, (ii) moderately-differentiated between $50-95 \%$, and (iii) poorly-differentiated between 5-49\%, and undifferentiated $<5 \%$. Evidence for lymphatic, venous and, perinureral invasion, and number of metastatic lymph nodes were recorded for colorectal cancers. TNM staging was recorded based on postsurgical findings.

\section{Genomic DNA extraction, EMAST and MSI analysis}

Serial $10 \mu \mathrm{m}$ sections of tissues were cut from formalin-fixed and paraffin-embedded tissues and mounted on glass slides. Microdissection was performed to extract genomic DNA from tumor and counterpart normal tissue. Genomic DNA from microdissected tissues was isolated using Qiagen DNeasy Blood and Tissue Kit (Qiagen, USA) following the manufacturer's instruction. Briefly, one thousand $\mu 1$ of xylene was added to each eppendorf tube containing microdissected tissue, and the tubes were centrifuged at 14,000 rpm for $5 \mathrm{~min}$ at room temperature (RT). After removing the supernatant, $1000 \mu \mathrm{l}$ of $100 \%$ ethanol was added, and all the tubes were centrifuged at 14,000 rpm for $5 \mathrm{~min}$ at RT. After removing the ethanol, the samples were incubated at $37^{\circ} \mathrm{C}$ for $15-25$ minutes until the ethanol was evaporated. Tissues were incubated in lysis buffer solution containing $20 \mu \mathrm{l}$ of proteinase $\mathrm{K}$ solution at $56^{\circ} \mathrm{C}$ overnight. On the following day, samples were treated with buffers according to the manufacturer's instructions, and were centrifuged at 8,000 rpm for $1 \mathrm{~min}$ twice and 14,000 rpm for $3 \mathrm{~min}$. After incubating at room temperature for $5 \mathrm{~min}$, DNA was extracted by centrifuging at $8,000 \mathrm{rpm}$ for $1 \mathrm{~min}$.

To determine EMAST, 5 polymorphic tetranucleotide markers (MYCL1, D9S242, D20S85, D8S321, and D20S82) were utilized. Genomic DNA extracted from tumors and counterpart normal tissues were PCR-amplified by specific primers for each tetranucleotide marker using Platinum ${ }^{\circledR}$ PCR Supermix (Invitrogen, USA) as per the manufacturer's protocol. The PCR products were used for DNA sequencing using an ABI 3700 analyzer to identify mutation at tetranucleotide repeats of each loci. Tumors showing frameshifts in at least 2 tetranucleotide markers were categorized as EMAST-positive tumors.

MSI analysis was performed using five mononucleotide markers (BAT-25, BAT-26, NR-21, $N R-24$, and MONO-27) and three dinucleotide markers (D2S123, D17S250, and D5S346) for MSI determination and two polymorphic pentanucleotide markers (Penta C and Penta D) for sample identification. We defined MSI-H when $>30 \%$ of examined loci showed frameshifts, MSI-L when $<30 \%$ but $>0 \%$ of examined loci had frameshifts, and MSS (microsatellite stable) when no instability was present at any of the loci tested. 


\section{Immunohistochemistry for hMSH3}

Slides containing colon tumor tissue were deparaffinized in xylene, and rehydrated in $100 \%$, $95 \%$, and $70 \%$ alcohols to water. The slides were immersed in sodium citrate buffer ( $\mathrm{pH} 6.0)$ and heated in a microwave for 10 minutes for antigen retrieval. Endogenous peroxidase activity was blocked by incubation with $3 \% \mathrm{H}_{2} \mathrm{O}_{2}$. Ten percent horse serum was added for 1 hour to block nonspecific protein binding. Rabbit hMSH3 antibody was incubated overnight at 1:800 dilution. Slides were then processed using a DAKO Signal Catalyzed Amplification System (DAKO Corp., Carpinteria, CA). Biotinylated anti-rabbit immunoglobulin $\mathrm{G}$ was added for 15 minutes, followed by incubation with peroxidase-labeled streptavidin for 15 minutes at room temperature. The sections were incubated with 3,3-diaminobenzidine tetrahydrochloride and $\mathrm{H}_{2} \mathrm{O}_{2}$ for 3 minutes, counterstained with hematoxylin, dehydrated in graded alcohols, cleared in xylene, and coverslipped. The percentage of blue basal nuclei stain among tumor cells was calculated as hMSH3 loss using a 100× objective lens. Nuclear heterogeneity was determined when there was both brown (stained) and blue (unstained) nuclear staining using a $400 \times$ objective lens. Slides were analyzed by a single pathologist who was unaware of the clinical, MSI, and EMAST results.

\section{Statistical analysis}

To study the statistical association between EMAST and other variables, continuous and categorical demographic data were compared by $t$-tests and chi-square tests, respectively. A $p$ value of less than 0.05 was considered statistically significant.

\section{RESULTS}

\section{The frequency of EMAST increases in more advanced colorectal neoplasms}

EMAST was determined when at least 2 loci showed frameshifts at tetranucleotide repeat loci compared to matched normal tissue. Overall, 50\% of tumors from 108 patients possessing 24 adenomas and 84 colorectal cancers were EMAST-positive. Additionally, by standardized MSI analysis, there were 6 MSI-H, 10 MSI-L, and 92 MSS colorectal neoplasms among the 108 tumors (Table 1). All 6 MSI-H (100\%) neoplasms were cancers, and all (100\%) were EMASTpositive, whereas 7 of 10 MSI-L neoplasms (70\%, all cancers) were EMAST-positive. All 24 adenomas were MSS.

To eliminate the possibility of MSI-H tumors cross-identifying EMAST tumors, we analyzed our cohort for EMAST after excluding the 6 MSI-H cases. Clinicopathological characteristics of 48 EMAST-positive tumor cases versus 54 EMAST-negative tumor cases are summarized in Table 1. There was no significant difference for location $(\mathrm{p}=0.638)$, shape $(0.536)$, or size $(\mathrm{p}=0.392)$ between EMAST-positive and EMAST-negative adenomas. The proportion of EMAST-positive neoplasms increased with the advancement of histology. Moderately differentiated adenocarcinomas (56.9\%) and poorly differentiated adenocarcinomas $(40.0 \%)$ showed higher frequency of EMAST than well differentiated adenocarcinomas $(12.5 \%)$ and adenomas (33.3\%) $(p=0.040)$. Individual details of the 40 EMAST-positive cancers are summarized in Table 2.

\section{Higher frequency of EMAST in ulcerated colorectal tumors}

EMAST-positive and -negative tumors cases were also categorized by endoscopic findings (Table 1). We found that downward growing ulcerated (depressed or excavated) tumors (52.3\%) showed a significantly higher frequency for EMAST-positivity than sessile (superficial or flat) tumors (44.0\%) and protruded (elevated or polypoid) tumors $(20.0 \%)$ $(p=0.049)$. In addition, of a total of 62 ulcerated cancers, regardless of growth patterns, 34 
(54.8\%) showed EMAST, whereas only 6 (37.5\%) of 16 non-ulcerated cancers showed EMAST.

\section{Relation of EMAST with tumor location and invasiveness of colorectal cancers}

There was no significant preference for location of EMAST-positive tumors, as $42.8 \%, 41.3 \%$, and $60.7 \%$ of EMAST was in proximal, distal, and rectal locations, respectively (Table 1). However, one EMAST locus, Mycll, showed a significantly higher frequency $(46.4 \%)$ of frameshifts in rectal tumors compared with proximal (21.4\%) and distal (19.6\%) colon tumors $(p=0.030)$. The other 4 EMAST loci tested did not show any difference in frameshift frequency relative to the location of the neoplasms.

There was no significant association between EMAST and tumor microinvasion (venous invasion, perineural invasion, and lymphatic invasion) among the 84 colorectal cancers. Venous invasion was noticed in 4 of 40 EMAST-positive cancers $(10.0 \%)$ and in 1 of 38 EMAST-negative cancers $(2.6 \%)$, respectively $(p=0.359)$. However, the EMAST locus Mycll was more frequently frameshifted in cancers with venous invasion $(80.0 \%)$ than cancers without venous invasion $(27.4 \%)(p=0.029)$. Additionally, perineural invasion was noticed in 6 of 40 EMAST-positive cancers (15.0\%) and in 4 of 38 EMAST-negative cancers (10.5\%), respectively ( $p=0.738)$. On the other hand, lymphatic invasion was noticed in 8 of 40 EMASTpositive cancers $(20.0 \%)$ and in 8 of 38 EMAST-negative cancers $(21.1 \%)$, respectively $(p=1.000)$. The EMAST marker D20S82 was less frequently observed in cancers with lymphatic invasions $(12.5 \%)$ than cancers without lymphatic invasion $(45.2 \%)(p=0.021)$.

None of the T, N, M stages correlated with the presence of EMAST. The frequency of EMAST was $40 \%$ in Tis, $50 \%$ in T1, 33.3\% in T2, 58.0\% in T3, and $42.9 \%$ in T4 ( $p=0.574)$. EMAST prevalence according to $\mathrm{N}$ staging was $51.3 \%$ in $\mathrm{N} 0,45.5 \%$ in $\mathrm{N} 1,58.8 \%$ in $\mathrm{N} 2(p=0.710)$. Additionally, there was no significant difference in EMAST frequency between cancers with metastasis $(54.5 \%)$ and without metastasis $(44.2 \%)(p=1.000)$.

\section{Loss of hMSH3 expression and heterogeneity}

Most tumors demonstrated nuclear heterogeneity by expressing both brown (positive) and blue (negative) nuclei upon hMSH3 immunohistochemical staining (Figure 2). This nuclear heterogeneity was noticed in 16 of 40 EMAST-positive cancers $(40.0 \%)$, whereas it was detected only in 5 of 38 EMAST-negative cancers $(13.2 \%)(p=0.010)$. Quantitatively, all tumors with $>3$ tetranucleotide defects showed significant hMSH3 loss (blue nuclei exceeding $75 \%$ of all stained basal nuclei in tumor cells), whereas this degree of hMSH3 loss was rare in tumors with no tetranucleotide defects (Figure 3).

\section{DISCUSSION}

In this study, we examined the frequency of EMAST in colorectal neoplasms and its linkage to clinicopathological variables, as there is no data for EMAST in this regard for CRC. We discovered that EMAST is less frequent in colorectal adenomas and well differentiated adenocarcinomas than in moderately differentiated and poorly differentiated adenocarcinomas, suggesting that EMAST is progressively acquired during the histological adenoma-carcinoma sequence, from adenoma to well-differentiated carcinomas to moderately and poorly differentiated carcinomas. CRC progresses genetically through at least two pathways involving genomic instability: chromosomal instability (CIN) and MSI, with the latter phenotype caused by loss of DNA MMR. Although it is still not clear whether EMAST just represents bystander mutations in a large subset of tumors with specific defects in DNA replication and repair, the lower EMAST prevalence in adenomas in our study suggests that the adenoma-carcinoma sequence is related to the development of EMAST. 
In defining MSI originally, ${ }^{1}$ some tumors were characterized as MSI-L and not associated with loss of DNA MMR, the cause of MSI-H. This definition is predicated on utilizing mono and dinucleotide markers, and not tetranucleotide markers. ${ }^{1}$ Longer repeats in microsatellites that frameshift are characteristically caused by loss of hMSH3 function, based on bacteria and gene data, as this DNA MMR protein helps repair longer insertion-deletion DNA loops during DNA replication. ${ }^{2}$ The finding of heterogenous expression of hMSH3 in CRC that are EMASTpositive seems to confirm hMSH3 as a potential cause of EMAST. Could tumor progression be associated with loss of hMSH3? This question has not been answered and may be somewhat difficult given the heterogenous nature of hMSH3 loss in colorectal tumors. ${ }^{15}$ Our finding strongly indicates that EMAST is an acquired feature of CRC. Additionally, our data showing nuclear heterogeneity for hMSH3 expression among EMAST-positive tumors, plus the quantitative association of the number of tetranucleotide frameshifts linked to hMSH3 loss strongly suggest that hMSH3 loss is linked to EMAST-positive neoplasm.

We found that EMAST is more frequent in colorectal tumors with ulcerated features. We speculate that EMAST association with ulcerated cancers may be a consequence of increased local inflammation. Colorectal tumors develop an increasing level of inflammatory infiltration during progression from adenoma to cancer, and would be highest in cancers in which mucosa is breached as in ulcerated cancers. Endoscopically, colon tumors grow in three different ways; upward, downward, and lateral. ${ }^{16}$ Of these three types, depressed lesions are most aggressive and inflammatory due to its ulceroinfiltrative nature. This is consistent with our result that tumors with downward growth showed higher prevalence of EMAST than upward or lateral growth tumors. In addition, cancers with ulceration showed higher prevalence of EMAST than cancers without ulceration.

It is possible that inflammation may drive EMAST. First, samples from inflamed colons from ulcerative colitis patients demonstrate MSI-L without major DNA MMR defects, but were not specifically tested for loss of hMSH3 expression. ${ }^{17}$ Second, in vitro experiments indicated that oxidative stress can "relax" or impair DNA MMR function, and can cause MSI. ${ }^{18,19}$ Thus, one hypothesis is that during the adenoma-carcinoma progression, EMAST is generated by progressive loss of hMSH3, for instance, due to inflammatory-induced impairment of this protein's function. As indicated in our study, hMSH3-positive and -negative cells were interspersed in most of the tumors, with nuclear heterogeneity being more common in EMASTpositive cancers than in -negative cancers. This suggests the possibility of hMSH3-negativity being a reversible trait as mentioned by Haugen et al. ${ }^{15}$ In our study, cancers with an ulcerative appearance showed a higher number of tetranucleotide defects, and all cancers with $>3$ tetranucleotide defects showed severe loss of hMSH3 expression. Our results are consistent with a hypothesis that the inflammatory process in ulceroinfiltrative cancers might be related to down-regulation of hMSH3 expression, and as a consequence demonstrate a higher number of tetranucleotide defects. In addition, recent evidence suggests EMAST is associated with increased numbers of inflammatory cells infiltrating a tumor. ${ }^{20}$

In regard to the invasiveness of cancers, there was no correlation between EMAST and TNM stages as well as with venous, perineural, or lymphatic invasions. Similarly, there was no significant difference in EMAST prevalence based on tumor location in the colon. This suggests that these parameters occur independent of EMAST, which is in contrast to classic MSI-H, which is associated with a right colon location, earlier cancer stage, and less tumor invasion. It is also possible that utilizing 2 of 5 teranucleotide frameshifts as the definition of EMAST might not count some EMAST tumors, as some have defined EMAST-positivity when 1 of 5 markers show a frameshift. In this study, if we defined EMAST as 1 of 5 defective tetranucleotide markers, the overall prevalence of EMAST increased from 50\% to $88.9 \%$ (96/108), a prevalence higher than in previous reports. ${ }^{14,15,20}$ Given the association of consistent hMSH3 loss with $>3$ tetranucleotide frameshifts in our study, a frequency higher 
than one tetranucleotide defect will likely be more appropriate to define EMAST as new data is produced. Despite this, it is still possible that some EMAST-positive tumors might have been ignored by using our definition of EMAST, since 5 of 54 EMAST-negative tumors showed hMSH3 heterogeneity by immunohistochemistry. A large-scale study may help determine the detection sensitivity of EMAST-positive tumors in relationship to hMSH3 expression.

In summary, EMAST is less frequent in colorectal adenomas, well differentiated adenocarcinomas, and colorectal cancers without ulcerations. EMAST is associated with nuclear heterogeneity of hMSH3 expression. These observations suggest that the EMAST phenotype is linked to adenoma-to-carcinoma morphology as well as the endoscopic appearance of ulceration, and nuclear hMSH3 expression.

\section{Acknowledgments}

The authors thank to Dr. Katsuya Miyai for analyzing the results of the hMSH3 immunohistochemical stains. This study was supported by Konkuk University, the United States Public Health Service (DK067287), the UCSD Digestive Diseases Research Development Center (DK080506), and the SDSU/UCSD Comprehensive Cancer Center Partnership (CA132379 and CA13238).

\section{REFERENCES}

1. Boland CR, Thibodeau SN, Hamilton SR, Sidransky D, Eshleman JR, Burt RW, Meltzer SJ, RodriguezBigas MA, Fodde R, Ranzani GN, Srivastava S. A National Cancer Institute Workshop on Microsatellite Instability for cancer detection and familial predisposition: development of international criteria for the determination of microsatellite instability in colorectal cancer. Cancer Res 1998;58:5248-5257. [PubMed: 9823339]

2. Grady WM, Carethers JM. Genomic and epigenetic instability in colorectal cancer pathogenesis. Gastroenterology 2008;135:1079-1099. [PubMed: 18773902]

3. Boland CR, Koi M, Chang DK, Carethers JM. The biochemical basis of microsatellite instability and abnormal immunohistochemistry and clinical behavior in Lynch syndrome: from bench to bedside. Fam Cancer 2008;7:41-52. [PubMed: 17636426]

4. Goel A, Arnold CN, Niedzwiecki D, Chang DK, Ricciardiello L, Carethers JM, Dowell JM, Wasserman L, Compton C, Mayer RJ, Bertagnolli MM, Boland CR. Characterization of sporadic colon cancer by patterns of genomic instability. Cancer Res 2003;63:1608-1614. [PubMed: 12670912]

5. Xu L, Chow J, Bonacum J, Eisenberger C, Ahrendt SA, Spafford M, Wu L, Lee SM, Piantadosi S, Tockman MS, Sidransky D, Jen J. Microsatellite instability at AAAG repeat sequences in respiratory tract cancers. Int J Cancer 2001;91:200-204. [PubMed: 11146445]

6. Ahrendt SA, Decker PA, Doffek K, Wang B, Xu L, Demeure MJ, Jen J, Sidransky D. Microsatellite instability at selected tetranucleotide repeats is associated with p53 mutations in non-small cell lung cancer. Cancer Res 2000;60:2488-2491. [PubMed: 10811129]

7. Danaee H, Nelson HH, Karagas MR, Schned AR, Ashok TD, Hirao T, Perry AE, Kelsey KT. Microsatellite instability at tetranucleotide repeats in skin and bladder cancer. Oncogene 2002;21:4894-4899. [PubMed: 12118368]

8. Catto JWF, Azzouzi AR, Amira N, Rehman I, Feeley KM, Cross SS, Fromont G, Sibony M, Hamdy FC, Cussenot O, Meuth M. Distinct patterns of microsatellite instability are seen in tumours of the urinary tract. Oncogene 2003;22:8699-8706. [PubMed: 14647464]

9. Burger M, Denzinger S, Hammerschmied CG, Tannapfel A, Obermann EC, Wieland WF, Hartmann A, Stoehr R. Elevated microsatellite alterations at selected tetranucleotides (EMAST) and mismatch repair gene expression in prostate cancer. J Mol Med 2006;84:833-841. [PubMed: 16924473]

10. Azzouzi AR, Catto JW, Rehman I, Larre S, Roupret M, Feeley KM, Cussenot O, Meuth M, Hamdy FC. Clinically localised prostate cancer is microsatellite stable. BJU Int 2007;99:1031-1035. [PubMed: 17233803] 
11. Choi YD, Choi J, Kim JH, Lee JS, Lee JH, Choi C, Choi HS, Lee MC, Park CS, Juhng SW, Nam JH. Microsatellite instability at a tetranucleotide repeat in type I endometrial carcinoma. J Exp Clin Cancer Res 2008;27:88. [PubMed: 19116039]

12. Kloor M, Schwitalle Y, von Knebel Doeberitz M, Wentzensen N. Tetranucleotide repeats in coding regions: no evidence for involvement in EMAST carcinogenesis. J Mol Med 2006;84:329-333. [PubMed: 16416315]

13. Jung B, Smith EJ, Doctolero RT, Gervaz P, Alonso JC, Miyai K, Keku T, Sandler RS, Carethers JM. Influence of target gene mutations on survival, stage and histology in sporadic microsatellite unstable colon cancers. Int J Cancers 2006;118:2509-2513.

14. Yamada K, Kanazawa S, Koike J, Sugiyama H, Xu C, Funahashi K, Boland CR, Koi M, Hemmi H. Microsatellite instability at tetranucleotide repeats in sporadic colorectal cancer in Japan. Oncol Rep 2010;23:551-561. [PubMed: 20043121]

15. Haugen AC, Goel A, Yamada K, Marra G, Nguyen TP, Nagasaka T, Kanazawa S, Koike J, Kikuchi Y, Zhong X, Arita M, Shibuya K, Oshimura M, Hemmi H, Boland CR, Koi M. Genetic instability caused by loss of MutS homologue 3 in human colorectal cancer. Cancer Res 2008;68:8465-8472. [PubMed: 18922920]

16. Kudo S, Takemura O, Ohtsuka K. Flat and depressed types of early colorectal cancers: from East to West. Gastrointest Endosc Clin N Am 2008;18:581-593. [PubMed: 18674705]

17. Brentnall TA, Crispin DA, Bronner MP, Cherian SP, Hueffed M, Rabinovitch PS, Rubin CE, Haggitt RC, Boland CR. Microsatellite instability in nonneoplastic mucosa from patients with chronic ulcerative colitis. Cancer Res 1996;56:1237-1240. [PubMed: 8640805]

18. Gasche C, Chang CL, Rhees J, Goel A, Boland CR. Oxidative stress increases frameshift mutations in human colorectal cancer cells. Cancer Res 2001;61:7444-7448. [PubMed: 11606378]

19. Chang CL, Marra G, Chauhan DP, Ha HT, Chang DK, Ricciardiello L, Randolph A, Carethers JM, Boland CR. Oxidative stress inactivates the DNA mismatch repair system. Am J Physiology Cell Physiol 2002;283:C148-C154.

20. Deveraj B, Lee A, Cabrera BL, Miyai K, Luo L, Ramamoorthy S, Keku T, Sandler RS, McGuire K, Carethers JM. Relationship of EMAST and microsatellite instability among patients with rectal cancer. J Gastrointest Surgery. 2010 in press. 


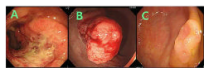

Figure 1. Endoscopic appearance of tumors

Tumors were subclassified into: (A) ulceroinfiltrative, depressed, or excavated tumors, (B) protruded, elevated, or polypoid tumors, and (C) sessile, superficial, or flat tumors. 


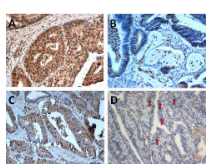

Figure 2. hMSH3 expression patterns by immunohistochemistry (A) An hMSH3-positive (brown nucleus staining) tumor. (B) An hMSH3-negative (blue nucleus staining) tumor. Most EMAST tumors showed (C) a mixed appearance with partial hMSH3 loss. (D) Heterogeneity was diagnosed when there is a nucleus showing both brown and blue staining in the nucleus (arrows). 


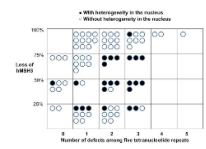

Figure 3. Degree of hMSH3 loss according to the number of tetranucleotide frameshifts among 78 cancers

Each black circle indicates a tumor with heterogeneity in the nucleus, whereas white circles indicate a tumor without heterogeneity. Tumors with $>3$ tetranucleotide defects showed the highest percentage of hMSH3 loss. 
Table 1

Correlation of EMAST and clinicopathological features

\begin{tabular}{|c|c|c|c|}
\hline & EMAST-positive tumors $(n=48)$ & EMAST-negative tumors $(n=54)$ & $p$-value \\
\hline Age (mean $\pm \mathrm{SD}$, year-old) & $60.31 \pm 12.97$ & $62.09 \pm 11.40$ & 0.462 \\
\hline Gender (male : female) & $29: 19$ & $36: 18$ & 0.542 \\
\hline Pathology & & & 0.040 \\
\hline Adenoma & $8(16.7 \%)$ & $16(29.6 \%)$ & \\
\hline Well differentiated adenocarcinoma & $1(2.1 \%)$ & $7(12.9 \%)$ & \\
\hline Moderately differentiated adenocarcinoma & $37(77.1 \%)$ & $28(51.9 \%)$ & \\
\hline Poorly differentiated adenocarcinoma & $2(4.2 \%)$ & $3(5.6 \%)$ & \\
\hline Endoscopic findings & & & 0.049 \\
\hline Upward growth (protruded, elevated, or polypoid) & $3(6.3 \%)$ & $12(22.2 \%)$ & \\
\hline Lateral growth (sessile, superficial, or flat) & $11(22.9 \%)$ & $14(25.9 \%)$ & \\
\hline $\begin{array}{l}\text { Downward growth (depressed, excavated, or } \\
\text { ulceroinfiltrative) }\end{array}$ & $34(70.8 \%)$ & $28(51.9 \%)$ & \\
\hline Location & & & 0.234 \\
\hline Proximal colon & $12(25.0 \%)$ & $16(29.6 \%)$ & \\
\hline Distal colon & $19(39.6 \%)$ & $27(50.0 \%)$ & \\
\hline Rectum & $17(35.4 \%)$ & $11(20.4 \%)$ & \\
\hline
\end{tabular}

EMAST, elevated microsatellite instability at selected tetranucleotide repeats. 


\begin{tabular}{|c|c|c|c|c|c|c|c|c|c|c|c|c|c|c|c|c|c|c|c|c|c|c|c|c|c|c|c|c|}
\hline 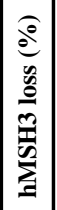 & 6 & $r$ & $\infty$ & $\infty$ & $=$ & 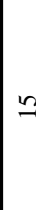 & $\stackrel{\infty}{-1}$ & 7 & & ? & $\approx$ & $m$ & $\stackrel{\infty}{\infty}$ & 8 & J & n & 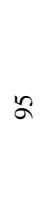 & $\alpha$ & 2 & 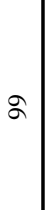 & a & $\mathrm{N}$ & $\vec{\sigma}$ & $\widetilde{\sigma}$ & 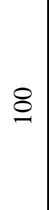 & 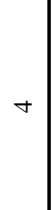 & 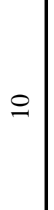 & \pm \\
\hline 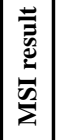 & $\sum_{\Sigma}^{n}$ & $\sum_{\Sigma}^{n}$ & $\frac{n}{\sum_{n}^{n}}$ & $\sum_{\Sigma}^{\tilde{n}}$ & $\sum_{\Sigma}^{n}$ & $\begin{array}{l}y \\
\Sigma \\
\Sigma\end{array}$ & $\sum_{n}^{n}$ & $\mid \begin{array}{l}y \\
z\end{array}$ & & $\frac{E}{2}$ & $\sum_{\Sigma}^{n}$ & $\sum_{\Sigma}^{n}$ & $\sum^{n}$ & $\sum_{\Sigma}^{n}$ & $\sum_{\Sigma}^{n}$ & $\frac{n}{\Sigma}$ & $\sum_{\bar{n}}^{\tilde{n}}$ & $\sum_{\Sigma}^{n}$ & $\begin{array}{l}n \\
\sum_{n}^{n}\end{array}$ & $\begin{array}{l}n \\
\sum_{n}^{n}\end{array}$ & $\sum_{n}^{n}$ & $\frac{\vec{\Delta}}{\vec{n}} \frac{1}{\Sigma}$ & $\begin{array}{l}\overrightarrow{1} \\
\frac{1}{2}\end{array}$ & $\frac{\vec{d}}{\sum^{n}}$ & $\frac{\overrightarrow{1}}{\sum_{n}^{n}}$ & $\begin{array}{l}\tilde{n}^{n} \\
n^{2}\end{array}$ & $\begin{array}{l}n \\
\sum_{2}^{n}\end{array}$ & $\mid \begin{array}{l}\tilde{n} \\
\sum_{n}^{n}\end{array}$ \\
\hline 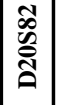 & 0 & & & & & & & $c$ & & & & & 0 & & & 0 & 0 & 0 & & 0 & & & & & & & 0 & 0 \\
\hline $\begin{array}{l}\bar{N} \\
\bar{D} \\
0 \\
\mathscr{0}\end{array}$ & & 0 & 0 & 10 & & 0 & & $c$ & & & 0 & $\bigcirc$ & 0 & 0 & 0 & & 0 & & 0 & & & 0 & 0 & 0 & & 0 & 0 & 0 \\
\hline 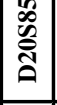 & & & & 0 & & & & & & & & & & & 0 & & & & & & 0 & 0 & 0 & & 0 & 0 & & 0 \\
\hline 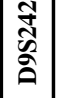 & & & & & 0 & & $c$ & & & & 0 & & & & & 0 & & 0 & 0 & & & & & 0 & 0 & 0 & 0 & \\
\hline $\begin{array}{l}\vec{\Xi} \\
\bar{z} \\
\Sigma\end{array}$ & 0 & 0 & 0 & & 0 & 0 & 0 & & & D & & 0 & & 0 & & & & & & $\bigcirc$ & 0 & & & & & & & \\
\hline$\frac{.}{\frac{\tilde{g}}{\pi}}$ & 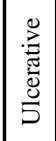 & 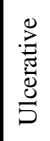 & 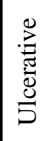 & 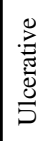 & 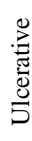 & 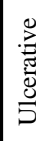 & \begin{tabular}{|l}
0 \\
$\frac{0}{2}$ \\
$\frac{5}{0}$ \\
0
\end{tabular} & 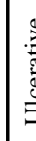 & & D. & 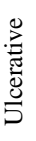 & 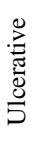 & 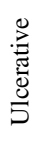 & 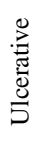 & 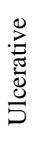 & 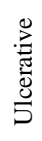 & 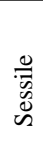 & 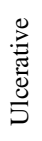 & 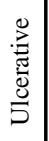 & 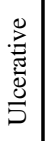 & 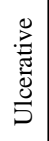 & 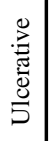 & 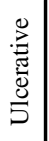 & 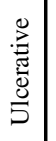 & 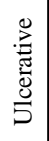 & $\begin{array}{l}\frac{0}{\bar{x}} \\
\bar{\nu} \\
\omega\end{array}$ & 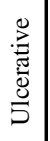 & 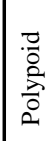 \\
\hline 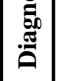 & $\hat{\Sigma}$ & 立 & $\hat{\Sigma}$ & 立 & 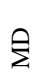 & 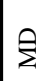 & $\xi$ & $\xi$ & & 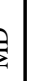 & $\stackrel{\partial}{\Sigma}$ & $\hat{\Sigma}$ & $\hat{2}$ & 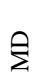 & 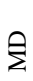 & 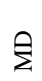 & 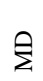 & 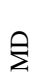 & $\mathrm{\Sigma}$ & 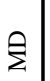 & 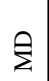 & 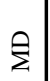 & $\mathrm{\Sigma}$ & $\stackrel{2}{2}$ & $\stackrel{\AA}{\Sigma}$ & $\xi$ & $\mathrm{\Sigma}$ & 立 \\
\hline$\dot{z}$ & ले & $\stackrel{\varrho}{=}$ & $\stackrel{\infty}{\infty}$ & ปิ & 三 & $\Sigma$ & 。 & in & & 6 & $\underline{\infty}$ & $\stackrel{\infty}{=}$ & $气$ & $=$ & 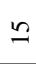 & 8 & $\tilde{\imath}$ & $n$ & $\stackrel{\Omega}{\Omega}$ & $\bar{\beth}$ & 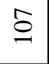 & ळे & ثे & $\Sigma$ & $\bar{\sigma}$ & $r$ & 余 & in \\
\hline
\end{tabular}




\begin{tabular}{|c|c|c|c|c|c|c|c|c|c|c|c|c|}
\hline & & & 0 & 0 & 0.0 & & & 0 & & & 0 & O \\
\hline & 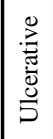 & 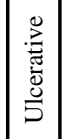 & 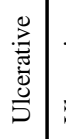 & 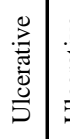 & 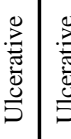 & 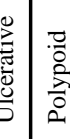 & 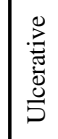 & 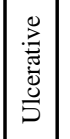 & 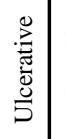 & 竞 & & \\
\hline قَّ & $\sum$ & 妾 & ह & $\frac{\partial}{\Sigma}$ & $\stackrel{े}{\Sigma}$ & $\frac{\partial}{\Sigma}$ & 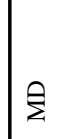 & $\frac{1}{\Sigma}$ & $\sum_{2}$ & 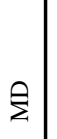 & 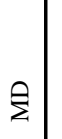 & $\frac{\partial}{\Sigma}$ \\
\hline & f & 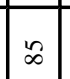 & 2 & $\stackrel{8}{\circ}$ & $\cong$ & $\sigma m$ & $\approx$ & $=$ & $n$ & $\hat{\lambda}$ & $F$ & $\exists$ \\
\hline
\end{tabular}

